PROCEEDINGS OF THE

AMERICAN MATHEMATICAL SOCIETY

Volume 135, Number 3, March 2007, Pages 705-711

S 0002-9939(06)08439-5

Article electronically published on October 2, 2006

\title{
(APD)-PROPERTY OF $C^{*}$-ALGEBRAS BY EXTENSIONS OF $C^{*}$-ALGEBRAS WITH (APD)
}

\author{
YIFENG XUE
}

(Communicated by David R. Larson)

\begin{abstract}
A unital $C^{*}$-algebra $\mathcal{A}$ is said to have the (APD)-property if every nonzero element in $\mathcal{A}$ has the approximate polar decomposition. Let $\mathcal{J}$ be a closed ideal of $\mathcal{A}$. Suppose that $\tilde{\mathcal{J}}$ and $\mathcal{A} / \mathcal{J}$ have (APD). In this paper, we give a necessary and sufficient condition that makes $\mathcal{A}$ have (APD). Furthermore, we show that if $\operatorname{RR}(\mathcal{J})=0$ and $\operatorname{tsr}(\mathcal{A} / \mathcal{J})=1$ or $\mathcal{A} / \mathcal{J}$ is a simple purely infinite $C^{*}$-algebra, then $\mathcal{A}$ has (APD).
\end{abstract}

\section{INTRODUCTION}

It is well known that every bounded linear operator on a Hilbert space has the polar decomposition, and moreover every nonzero element in a Von Neumann algebra has the polar decomposition. This property also holds for certain $A W^{*_{-}}$ algebras (cf. [4]). For a $C^{*}$-algebra $\mathcal{A}, a \in \mathcal{A} \backslash\{0\}$ can be written as $a=v|a|$, where $|a|=\left(a^{*} a\right)^{1 / 2}$ and $v$ is a partial isometry in $\mathcal{A}^{\prime \prime}$ which is not in $\mathcal{A}$ generally. But if $a$ is well supported, i.e., there is a projection $p \in \mathcal{A}$ such that $a=a p$ and $|a|$ is invertible in $p \mathcal{A} p$ with the inverse $a_{0}$ (cf. [1, Definition 6.5.3]), then $v=a a_{0}$ is a partial isometry in $\mathcal{A}$ and $a=v|a|, a\left(a_{0} v\right) a=a$, that is, $a$ has the polar decomposition in $\mathcal{A}$. This leads us to seek a suitable definition so that every nonzero element in a $C^{*}$-algebra has a kind of polar decomposition. An approximate version of the polar decompositon of an element is as follows.

Let $\mathcal{A}$ be a unital $C^{*}$-algebra and $a \in \mathcal{A} /\{0\}$. We call $a$ the approximate polar decomposition (for short (APD)) if for any $\epsilon>0$, there is a partial isometry $v$ in $\mathcal{A}$ such that $\|a-v|a|\|<\epsilon$. We will say $\mathcal{A}$ has the (APD)-property if every nonzero element in $\mathcal{A}$ has the (APD). If $\mathcal{A}$ is nonunital and $\widetilde{A}$ has the (APD)-property, we also say $\mathcal{A}$ has the (APD)-property, where $\widetilde{\mathcal{A}}$ is the unitarization of $\mathcal{A}$.

For a $C^{*}$-algebra $\mathcal{A}$, let $\operatorname{Ws}(\mathcal{A})$ denote the set of all well-supported elements in $\mathcal{A}$ and put $\operatorname{Rg}(\mathcal{A})=\{a \in \mathcal{A} \backslash\{0\} \mid a \in a \mathcal{A} a\}$. Denote by Pis $(\mathcal{A})$ the set of all partial isometries and $\operatorname{set} \operatorname{ext}(\mathcal{A})=\left\{v \in \operatorname{Pis}(\mathcal{A}) \mid\left(1-v^{*} v\right) \mathcal{A}\left(1-v v^{*}\right)=\{0\}\right\}$. Recall from [3] (resp. 6]) that a unital $C^{*}$-algebra $\mathcal{A}$ is said to be (resp. have) extremally rich (resp. (WS)-property) if $\mathcal{A}_{q}^{-1}=\{x v y \mid v \in \operatorname{ext}(\mathcal{A}), x, y$ invertible in $\mathcal{A}\}$ (resp.

Received by the editors February 15, 2005 and, in revised form, July 15, 2005.

2000 Mathematics Subject Classification. Primary 46L05, 46L85, 46L80.

Key words and phrases. Approximate polar decomposition, generalized inverse, (WS)property.

This research was supported by the Natural Science Foundation of China and the Foundation of CSC. 
$\mathrm{Ws}(\mathcal{A})$ ) is dense in $\mathcal{A}$. If $\mathcal{A}$ is nonunital and $\overline{\widetilde{\mathcal{A}}_{q}^{-1}}$ (resp. $\left.\overline{\mathrm{Ws}(\widetilde{\mathcal{A}})}\right)=\widetilde{\mathcal{A}}$, then we call $\mathcal{A}$ extremally rich (resp. has (WS)).

Let $\mathcal{A}$ be a unital $C^{*}$-algebra. Since $\mathcal{A}_{q}^{-1} \subset \operatorname{Rg}(\mathcal{A})=\operatorname{Ws}(\mathcal{A})$ and $\mathcal{A}$ has $(\mathrm{APD})$ iff $\mathcal{A}$ has (WS) (Proposition 2.1), it follows that if $\mathcal{A}$ is of real rank zero or extremally rich, $\mathcal{A}$ has (APD) (cf. [1, Proposition 6.5.4]). Moreover, by [6], when $\mathcal{A}$ has (APD), the real rank of $\mathcal{A}$ is no more than one, and if $\mathcal{J}$ is a hereditary $C^{*}$-subalgebra of $\mathcal{A}$, then $\widetilde{J}$ has (APD). In particular, if $\mathcal{J}$ is a closed ideal of $\mathcal{A}$, then $\mathcal{A} / \mathcal{J}$ also has (APD).

In order to enlarge this kind of $C^{*}$-algebra, we consider whether the (APD)property can pass the extensions of $C^{*}$-algebras, that is, let $\mathcal{J}$ be a closed ideal of a unital $C^{*}$-algebra $\mathcal{A}$ and assume that $\mathcal{J}$ and $\mathcal{B}=\mathcal{A} / \mathcal{J}$ have (APD). Then under what conditions does $\mathcal{A}$ have (APD)? This is the first motivation to do this work. Let $\mathcal{J}$ be a nonunital separable simple $C^{*}$-algebra with real rank zero. It has been known that in many cases, the multiplier algebra $M(\mathcal{J})$ is not extremally rich even if $M(\mathcal{J}) / \mathcal{J}$ is extremally rich (cf. 8) ). This encourages us to seek a weaker condition than extremal richness on $M(\mathcal{J})$. The (APD)-property of $M(\mathcal{J})$ could be considered, and it will appear in another paper. But this consideration becomes our other motivation to do this work.

In what follows, $\mathcal{A}$ is a unital $C^{*}$-algebra, $\mathcal{J}$ is a closed ideal of $\mathcal{A}$ and let $\pi: \mathcal{A} \rightarrow \mathcal{A} / \mathcal{J}=\mathcal{B}$ be the canonical homomorphism. Assume that $\mathcal{J}$ and $\mathcal{B}$ have (APD). The following theorem gives a complete answer when $\mathcal{A}$ has (APD).

Theorem 1.1. Assume that $\mathcal{J}$ and $\mathcal{B}$ have (APD). Then $\mathcal{A}$ has (APD) iff following two conditions are satisfied:

(1) For each $v \in \operatorname{Ais}(\mathcal{B})$ there is $w \in \operatorname{Pis}(\mathcal{A})$ such that $\pi(w) v^{*} v=v$ and

(2) $\operatorname{Pis}(\mathcal{A})+\mathcal{J} \subset \overline{\mathrm{Ws}(\mathcal{A})}$,

where $\operatorname{Ais}(\mathcal{B})=\left\{v \in \mathrm{Pis}(\mathcal{B})|b=v| b \mid \in \mathrm{Ws}_{0}(\mathcal{B})\right\}$ and $\mathrm{Ws}_{0}(\mathcal{B}) \subset \mathrm{Ws}(\mathcal{B})$ such that $\mathrm{Ws}_{0}(\mathcal{B})$ is dense in $\mathcal{B}$.

From Theorem 1.1, we can obtain an interesting result: if $\mathcal{J}$ is of real rank zero and $\mathcal{B}$ is of stable rank one or a simple purely infinite $C^{*}$-algebra, $\mathcal{A}$ has (APD).

\section{Preliminaries}

Let $\mathcal{E}$ be a $C^{*}$-algebra. According to [5], $a \in \operatorname{Rg}(\mathcal{E})$ iff 0 is an isolated point of $\sigma\left(a^{*} a\right)$ (or $0 \notin \sigma\left(a^{*} a\right)$ if $\mathcal{E}$ is unital) or iff $|a| \in \operatorname{Rg}(\mathcal{E})$ or iff there is a unique element $d \in \mathcal{E}$ such that $a d a=a, d a d=d,(a d)^{*}=a d,(d a)^{*}=d a$. The element $d$ is called the generalized inverse of $a$; we denote it by $a^{+}$. Consequently, if $a \in \operatorname{Rg}(\mathcal{E})$ and put $v=a|a|^{+}, p=|a|^{+}|a|$, then $a p=a, a=v|a|, p|a|=|a|$, that is, $a$ has the polar decompositon and $|a|$ is invertible in $p \mathcal{E} p$ with the inverse $|a|^{+}$. Thus, $\mathrm{Ws}_{\mathrm{s}}(\mathcal{E})=\operatorname{Rg}(\mathcal{E})$. Moreover, we have:

Proposition 2.1. Let $\mathcal{E}$ be a unital $C^{*}$-algebra. Then $\mathcal{E}$ has (APD) iff $\mathcal{E}$ has (WS).

Proof. If $\mathcal{E}$ has (APD), then for any $a \in \mathcal{E} \backslash\{0\}$ and any $\epsilon \in(0,1)$, there is $v \in$ $\operatorname{Pis}(\mathcal{E})$ such that $\|a-v|a|\|<\frac{\epsilon}{2}$. Put $a_{\epsilon}=v\left(|a|+\frac{\epsilon}{2}\right)$. Then $a_{\epsilon} \in \operatorname{Rg}(\mathcal{E})$ $\left(a_{\epsilon}\left(|a|+\frac{\epsilon}{2}\right)^{-1} v^{*} a_{\epsilon}=a_{\epsilon}\right)$ and $\left\|a-a_{\epsilon}\right\|<\epsilon$, i.e., $\overline{\mathrm{Ws}(\mathcal{E})}=\mathcal{A}$.

Now assume that $\mathcal{E}$ has (WS). Note that by [7, Lemma 2.5.11], there is $\delta>0$ such that $\left\|x_{1}^{1 / 2}-x_{2}^{1 / 2}\right\|<\frac{\epsilon}{2}$ whenever $x_{i} \in \mathcal{E}_{+}$with $\left\|x_{i}\right\| \leq 1$ and $\left\|x_{1}-x_{2}\right\|<\delta, i=1,2$.

Let $b \in \mathcal{E}$ with $\|b\|=\frac{1}{2}$. Choose $b_{\epsilon} \in \operatorname{Ws}(\mathcal{E})$ such that $\left\|b-b_{\epsilon}\right\|<\min \left\{\frac{2 \delta}{3}, \frac{\epsilon}{2}\right\}$. 
Then $\left\|b_{\epsilon}\right\| \leq 1$ and $\left\||b|^{2}-\left|b_{\epsilon}\right|^{2}\right\|<\delta$. It follows that $\left\||b|-\left|b_{\epsilon}\right|\right\|<\frac{\epsilon}{2}$. Let $b_{\epsilon}=v_{\epsilon}\left|b_{\epsilon}\right|$ be the polar decomposition. We have $\left\|b-v_{\epsilon}|b|\right\|<\epsilon$. For any $a \in \mathcal{E} \backslash\{0\}$, put $b=\frac{a}{2\|a\|}$. Then $\left\|a-v_{\epsilon}|a|\right\|<2\|a\| \epsilon$. So, $\mathcal{E}$ has (APD).

Let $\mathcal{E}$ be a $C^{*}$-algebra with unit 1 . We write $U(\mathcal{E})$ to denote the unitary group and $U_{0}(\mathcal{E})$ to denote the connected component of 1 in $U(\mathcal{E})$. Recall that $\mathcal{E}$ is of stable rank one, denoted by $\operatorname{tsr}(\mathcal{E})=1$ (resp. real rank zero, denoted by $\operatorname{RR}(\mathcal{E})=0$ ), if every (resp. self-adjoint) element in $\mathcal{E}$ can be approximated by an invertible (resp. self-adjoint) element in $\mathcal{E}$. If $\mathcal{E}$ is nonunital and $\widetilde{\mathcal{E}}$ is of stable rank one (resp. real rank zero), then we say $\operatorname{tsr}(\mathcal{E})=1($ resp. $\operatorname{RR}(\mathcal{E})=0)$.

Let $p, q$ be two nonzero projections in a $C^{*}$-algebra $\mathcal{E}$ and $a, b \in \mathcal{E}$. Set

$$
p \mathcal{E} q=\{p x q \mid x \in \mathcal{E}\}, \quad \mathrm{Ws}(p \mathcal{E} q)=\left\{a \in p \mathcal{E} q \mid a^{+} \text {exists, } q a^{+}=a^{+} p=a^{+}\right\} .
$$

Write

$$
\begin{gathered}
a_{11}=q a p, a_{12}=q a(1-p), a_{21}=(1-q) a p, a_{22}=(1-q) a(1-p), \\
b_{11}=p a q, b_{12}=p b(1-q), b_{21}=(1-p) b q, b_{22}=(1-p) b(1-q) .
\end{gathered}
$$

Then $a=a_{11}+a_{12}+a_{21}+a_{22}, b=b_{11}+b_{12}+b_{21}+b_{22}$. For convenience, we write $a$ (resp. $b$ ) as a matrix $a=\left[\begin{array}{ll}a_{11} & a_{12} \\ a_{21} & a_{22}\end{array}\right]$ (resp. $b=\left[\begin{array}{ll}b_{11} & b_{12} \\ b_{21} & b_{22}\end{array}\right]$ ). It is easy to check that

$$
b a=\left[\begin{array}{ll}
b_{11} a_{11}+b_{12} a_{21} & b_{11} a_{12}+b_{12} a_{22} \\
b_{21} a_{11}+b_{22} a_{21} & b_{21} a_{12}+b_{22} a_{22}
\end{array}\right] .
$$

Using the same method as in the proof of [7, Lemma 3.5.1], we have:

Lemma 2.2. Let $p, q$ be two nonzero projections in the $C^{*}$-algebra $\mathcal{E}$ and $a \in \mathcal{E}$. Put $x=$ paq and $\operatorname{Her}(|x|)=\overline{|x| \mathcal{E}|x|}$. Let $x=v|x|$ be the polar decomposition of $x$ in $\mathcal{E}^{\prime \prime}$. Then $p v=v q=v, b v^{*} v=b$ and $v b \in \mathcal{E}$ for every $b \in \operatorname{Her}(|x|)$.

The following corollary slightly improves [1, Proposition 6.5.4, Theorem 6.5.5].

Corollary 2.3. Let $p, q$ be two nonzero projections in the $C^{*}$-algebra $\widetilde{\mathcal{E}}$ and $a \in \mathcal{E}$. Assume that $x=p a q \neq 0$ and $\operatorname{RR}(\operatorname{Her}(|x|))=0$. Then for any $\epsilon>0$, there is $y \in \mathrm{Ws}(p \mathcal{E} q)$ such that $\|x-y\|<\epsilon$.

Proof. Let $x=v|x|$ be the polar decomposition in $\mathcal{E}^{\prime \prime}$ and put $p_{x}=v^{*} v$. Then by Lemma 2.2 $p v=v q=v, p_{x} b=b p_{x}=b$ and $v b \in \mathcal{E}$ for any $b \in \operatorname{Her}(|x|)$.

Since RR $(\operatorname{Her}(|x|))=0)$, it follows from [2, Theorem 2.6] that there are $\lambda_{1}, \cdots$, $\lambda_{s} \in \mathbb{R} \backslash\{0\}$ and mutually orthogonal projections $q_{1}, \cdots, q_{s}$ in $\operatorname{Her}(|x|)\left(q_{i} \leq q\right)$ such that $\left\||x|-\sum_{i=1}^{s} \lambda_{i} q_{i}\right\|<\epsilon$. Set $y=v\left(\sum_{i=1}^{s} \lambda_{i} q_{i}\right), y_{0}=\left(\sum_{i=1}^{s} \lambda_{i}^{-1} q_{i}\right) v^{*}$. Then $y, y_{0} \in \mathcal{E},\|x-y\|<\epsilon$ and $y_{0}=y^{+}$and moreover, $p y=y q=y, q y_{0}=y_{0} p=y_{0}$, i.e., $y \in \operatorname{Ws}(p \mathcal{E} q)$.

Proposition 2.4. If $\mathcal{J}$ is of real rank zero, then $\operatorname{Pis}(\mathcal{A})+\mathcal{J} \subset \overline{\mathrm{Ws}(\mathcal{A})}$.

Proof. Let $v$ be a partial isometry in $\mathcal{A}$. Put $p=v^{*} v, q=v v^{*}$ and

$$
\begin{array}{ll}
b_{11} & =q(v+k) p=q v\left(p+v^{*} k\right) p, \quad b_{12}=q(v+k)(1-p)=q k(1-p), \\
b_{21} & =(1-q)(v+k) p=(1-q) k p, \quad b_{22}=(1-q)(v+k)(1-p)=(1-q) k(1-p) .
\end{array}
$$

Since for any nonzero projections $p_{1}, p_{2} \in \mathcal{A}$ and any nonzero element $x \in p_{1} \mathcal{J} p_{2}$, $\operatorname{Her}(|x|)$ is a hereditary $C^{*}$-subalgebra of $\mathcal{J}$, it follows from [2, Corollary 2.8] and Corollary 2.3 that $\operatorname{Ws}\left(p_{1} \mathcal{J} p_{2}\right)$ is dense in $p_{1} \mathcal{J} p_{2}$. Noting that $\operatorname{RR}(p \mathcal{J} p)=$ 
$\operatorname{RR}(\mathcal{J})=0$ and $\operatorname{Her}\left(\left|p+v^{*} k p\right|\right)$ is a hereditary $C^{*}$-subalgebra of $p \widetilde{\mathcal{J}} p=\widetilde{p \mathcal{J}} p$, we have $\operatorname{RR}\left(\operatorname{Her}\left(\left|p+v^{*} k p\right|\right)\right)=0$ by [2, Corollary 2.8], and hence there is $k_{11} \in$ $\mathrm{Ws}(p \widetilde{\mathcal{J}} p)$ such that $\left\|p+v^{*} k-k_{11}\right\|<\frac{\epsilon}{4}$ by Corollary 2.3. Put $a_{11}=v k_{11}$. Then $a_{11} \in \operatorname{Ws}(q \mathcal{A} p)$ for $a_{11}^{+}=k_{11}^{+} v^{*}$ satisfying $p a_{11}^{+}=a_{11}^{+} q=a_{11}^{+}$. If we write

$$
v+k=\left[\begin{array}{ll}
b_{11} & b_{12} \\
b_{21} & b_{22}
\end{array}\right], \quad x_{0}=\left[\begin{array}{ll}
a_{11} & b_{12} \\
b_{21} & b_{22}
\end{array}\right],
$$

then $\left\|v+k-x_{0}\right\|<\frac{\epsilon}{4}$. Note that $x_{0}$ can be decomposed as

$$
x_{0}=\left[\begin{array}{cc}
q & 0 \\
b_{21} a_{11}^{+} & 1-q
\end{array}\right]\left[\begin{array}{cc}
a_{11} & \left(q-a_{11} a_{11}^{+}\right) b_{12} \\
b_{21}\left(p-a_{11}^{+} a_{11}\right) & b_{22}-b_{21} a_{11}^{+} b_{12}
\end{array}\right]\left[\begin{array}{cc}
p & a_{11}^{+} b_{12} \\
0 & 1-p
\end{array}\right] .
$$

Now put $b_{12}^{\prime}=\left(q-a_{11} a_{11}^{+}\right) b_{12}, \quad b_{21}^{\prime}=b_{21}\left(p-a_{11}^{+} a_{11}\right), b_{22}^{\prime}=b_{22}-b_{21} a_{11}^{+} b_{12}$. Then there exist $a_{12} \in \mathrm{Ws}\left(\left(q-a_{11} a_{11}^{+}\right) \mathcal{J}(1-p)\right), a_{21} \in \mathrm{Ws}\left((1-q) \mathcal{J}\left(p-a_{11}^{+} a_{11}\right)\right)$ such that $\left\|b_{12}^{\prime}-a_{12}\right\|$ and $\left\|b_{21}^{\prime}-a_{21}\right\|$ are all less than $\frac{\epsilon}{4\left\|1+b_{21} a_{11}^{+}\right\|\left\|1+a_{11}^{+} b_{12}\right\|}$. Put $x_{1}=\left[\begin{array}{ll}a_{11} & a_{12} \\ a_{21} & b_{22}^{\prime}\end{array}\right]$. Then by (2.1),

$$
\left\|x_{0}-\left[\begin{array}{cc}
q & 0 \\
b_{21} a_{11}^{+} & 1-q
\end{array}\right] x_{1}\left[\begin{array}{cc}
p & a_{11}^{+} b_{12} \\
0 & 1-p
\end{array}\right]\right\|<\frac{\epsilon}{2} .
$$

Decompose $x_{1}$ as

$$
x_{1}=\left[\begin{array}{cc}
q & 0 \\
b_{22}^{\prime} a_{12}^{+} & 1-q
\end{array}\right]\left[\begin{array}{cc}
a_{11} & a_{12} \\
a_{21} & d
\end{array}\right]\left[\begin{array}{cc}
p & a_{21}^{+} b_{22}^{\prime}\left(1-p-a_{12}^{+} a_{12}\right) \\
0 & 1-p
\end{array}\right],
$$

where $d=\left(1-q-a_{21} a_{21}^{+}\right) b_{22}^{\prime}\left(1-p-a_{12}^{+} a_{12}\right)$. Pick

$$
a_{22} \in \mathrm{Ws}\left(\left(1-q-a_{21} a_{21}^{+}\right) \mathcal{J}\left(1-p-a_{12}^{+} a_{12}\right)\right)
$$

such that

$$
\left\|d-a_{22}\right\|<\frac{\epsilon}{4\left\|1+b_{21} a_{11}^{+}\right\|\left\|1+a_{11}^{+} b_{12}\right\|\left\|1+b_{22}^{\prime} a_{12}^{+}\right\|\left\|1+a_{21}^{+} b_{22}^{\prime}\left(1-p-a_{12} a_{12}^{+}\right)\right\|} .
$$

Set $x_{2}=\left[\begin{array}{ll}a_{11} & a_{12} \\ a_{21} & a_{22}\end{array}\right]$ and

$$
x=\left[\begin{array}{cc}
q & 0 \\
b_{21} a_{11}^{+} & 1-q
\end{array}\right]\left[\begin{array}{cc}
q & 0 \\
b_{22}^{\prime} a_{12}^{+} & 1-q
\end{array}\right] x_{2}\left[\begin{array}{cc}
p & a_{21}^{+} b_{22}^{\prime}\left(1-p-a_{12}^{+} a_{12}\right) \\
0 & 1-p
\end{array}\right]\left[\begin{array}{cc}
p & a_{11}^{+} b_{12} \\
0 & 1-p
\end{array}\right] .
$$

Then $\|v+k-x\|<\epsilon$ by (2.2). Noting that $a_{11}^{*} a_{12}=a_{11}^{*}\left(q-a_{11} a_{11}^{+}\right) a_{12}=0$ and $a_{21}^{*} a_{22}=0$, we get that

$$
x_{2}^{*} x_{2}=\left[\begin{array}{cc}
a_{11}^{*} a_{11}+a_{21}^{*} a_{21} & 0 \\
0 & a_{12}^{*} a_{12}+a_{22}^{*} a_{22}
\end{array}\right] .
$$

But we also have $\left(a_{11}^{*} a_{11}\right)\left(a_{21}^{*} a_{21}\right)=0,\left(a_{12}^{*} a_{12}\right)\left(a_{22}^{*} a_{22}\right)=0$, so

$$
\sigma\left(x_{2}^{*} x_{2}\right)=\sigma\left(a_{11}^{*} a_{11}\right) \cup \sigma\left(a_{12}^{*} a_{12}\right) \cup \sigma\left(a_{21}^{*} a_{21}\right) \cup \sigma\left(a_{22}^{*} a_{22}\right) .
$$

This indicates that $x_{2} \in \mathrm{Ws}(\mathcal{A})$. Therefore, $x \in \mathrm{Ws}(\mathcal{A})$.

The following lemma comes from [7, Lemma 2.5.1, Lemma 2.5.4].

Lemma 2.5. Let $p$ be a projection in a $C^{*}$-algebra $\mathcal{E}$.

(1) If there is a projection $q \in \mathcal{E}$ such that $\|p-q\|<1$, then there is a unitary element $u \in \mathcal{E}$ such that $q=u p u^{*}$ and $\|1-u\|<\sqrt{2}\|p-q\|$.

(2) If there is an $a \in \mathcal{E}_{\text {sa }}$ such that $\|a-p\|<\frac{1}{2}$, then there is a projection $q$ in the $C^{*}$-subalgebra generated by a such that $\|p-q\|<2\|a-p\|$. 


\section{Proof of Theorem 1.1}

Proof of Theorem 1.1. $(\Leftarrow)$ Let $x \in \mathcal{A}$. Then for any $\epsilon>0$, there is $y \in \mathrm{Ws}_{0}(\mathcal{B})$ such that $\|\pi(x)-y\|<\frac{\epsilon}{3}$. Let $y=v|y|, v \in \operatorname{Ais}(\mathcal{B})$. By hypotheses, we have a partial isometry $w \in \mathcal{A}$ such that $\pi(w) v^{*} v=v$. Pick $a \in \mathcal{A}_{+}$with $\pi(a)=|y|$. Noting that $v^{*} v|y|=|y|$, we have $\pi(w a)=\pi(w) v^{*} v|y|=v|y|=y$. Put $x_{0}=$ $w\left(a+\frac{\epsilon}{3}\right)$. Then $\left\|\pi\left(x-x_{0}\right)\right\|<\frac{2 \epsilon}{3}$ and $x_{0} \in \mathrm{Ws}(\mathcal{A})$. Thus, we can find an element $k \in \mathcal{J}$ such that $\left\|x-x_{0}-k\right\|<\frac{2 \epsilon}{3}$. Applying Condition (2) to $w+k\left(a+\frac{\epsilon}{3}\right)^{-1}$, we obtain $z_{\epsilon} \in \operatorname{Ws}(\mathcal{A})$ such that

$$
\left\|w+k\left(a+\frac{\epsilon}{3}\right)^{-1}-z_{\epsilon}\right\|<\frac{\epsilon}{3\left\|a+\frac{\epsilon}{3}\right\|} .
$$

Put $x_{\epsilon}=z_{\epsilon}\left(a+\frac{\epsilon}{3}\right)$. Then $x_{\epsilon} \in \mathrm{Ws}(\mathcal{A})$ and $\left\|x-x_{\epsilon}\right\|<\epsilon$.

$(\Rightarrow)$ Let $v$ be in $\operatorname{Ais}(\mathcal{B})$. Then there is $x \in \mathrm{Ws}(\mathcal{A})$ such that $\|\pi(x)-v\|<\frac{1}{100}$. Let $x=u|x|$ be the polar decomposition and set $p^{\prime}=\pi\left(u^{*} u\right), q^{\prime}=\pi\left(u u^{*}\right), p=$ $v^{*} v, q=v v^{*}, b=\pi(|x|)$. Simple computation shows that

$$
\left\|p-b^{2}\right\|<\frac{3}{100},\left\|\left(1-p^{\prime}\right) p\right\|<\frac{3}{100},\left\|q-\pi(u) p \pi\left(u^{*}\right)\right\|<\frac{6}{100} .
$$

Note that $\|b\|<2$. Thus, from (3.1), we get that

$$
\|p b-b p\|<\frac{6\|b\|}{100}<\frac{12}{100}, \quad\|p b p b p-p\|<\frac{27}{100},
$$

so that $\|p b p-p\|<\frac{27}{100}$ by spectral theorem. Furthermore, $\|b p-p\|<\frac{39}{100}$ and $\|v-\pi(u) p\|=\|(v-\pi(u) b) p-\pi(u)(p-b p)\|<\frac{40}{100}$.

Now by (3.1), $\left\|p-p^{\prime} p p^{\prime}\right\|<\frac{6}{100}$. Thus, by Lemma 2.5(2), there are a projection $p_{0}^{\prime} \in p^{\prime} \mathcal{B} p^{\prime}$ and a unitary element $v_{0} \in U_{0}(\mathcal{B})$ such that

$$
\left\|p-p_{0}^{\prime}\right\|<2\left\|p-p^{\prime} p p^{\prime}\right\|<\frac{12}{100},\left\|1-v_{0}\right\|<\frac{12 \sqrt{2}}{100} \text { and } p=v_{0}^{*} p_{0}^{\prime} v_{0} .
$$

Choose $u_{0} \in U_{0}(\mathcal{A})$ such that $\pi\left(u_{0}\right)=v_{0}$. Put $w_{0}=u u_{0}$. Then $w_{0} \in \operatorname{Pis}(\mathcal{A})$ and $p \leq v_{0}^{*} p^{\prime} v_{0}=\pi\left(w_{0}^{*} w_{0}\right)$. From (3.1), we get that

$$
\begin{gathered}
\left\|q-\pi\left(w_{0}\right) p \pi\left(w_{0}^{*}\right)\right\|=\left\|q-\pi(u) p_{0}^{\prime} \pi\left(u^{*}\right)\right\| \\
\quad \leq\left\|q-\pi(u) p \pi\left(u^{*}\right)\right\|+\left\|p-p_{0}^{\prime}\right\|<\frac{18}{100} .
\end{gathered}
$$

Since $\pi\left(w_{0}\right) p \pi\left(w_{0}^{*}\right)$ is a projection, it follows from Lemma 2.5) (1) that there is $v_{1} \in U_{0}(\mathcal{B})$ with $\left\|1-v_{1}\right\|<\frac{18 \sqrt{2}}{100}$ such that $q=v_{1} \pi\left(w_{0}\right) p \pi\left(w_{0}^{*}\right) v_{1}^{*}$. Pick $u_{1} \in U(\mathcal{A})$ with $\pi\left(u_{1}\right)=v_{1}$ and set $w_{1}=u_{1} w_{0}$. Then $w_{1} \in \operatorname{Pis}(\mathcal{A})$ and $q=\pi\left(w_{1}\right) p \pi\left(w_{1}^{*}\right)$. Therefore, $q \pi\left(w_{1}\right)=\pi\left(w_{1}\right) p$ and $v^{*} \pi\left(w_{1}\right)$ is unitary in $p \mathcal{B} p$. Since

$$
\begin{aligned}
\left\|p-v^{*} \pi\left(w_{1}\right) p\right\| & \leq\left\|v-q \pi\left(w_{1}\right) p\right\|=\left\|v-\pi\left(w_{1}\right) p\right\| \\
& \leq\|v-\pi(u) p\|+\left\|\left(v_{1}-1\right) \pi(u) v_{0} p\right\|+\left\|\pi(u)\left(v_{0}-1\right) p\right\| \\
& <\frac{40}{100}+\frac{12 \sqrt{2}}{100}+\frac{18 \sqrt{2}}{100}<1,
\end{aligned}
$$

we have $v^{*} \pi\left(w_{1}\right) p+1-p \in U_{0}(\mathcal{B})$. Consequently, there is $u_{2} \in U(\mathcal{A})$ such that $\pi\left(u_{2}\right)=v^{*} \pi\left(w_{1}\right) p+1-p$. So, $p \pi\left(u_{2}\right)=\pi\left(u_{2}\right) p$. Put $w=w_{1} u_{2} *$. Then $w \in$ Pis $(\mathcal{A})$ and $v=\pi(w) p$. This proves Condition (1). Condition (2) is obvious since $\overline{\mathrm{Ws}(\mathcal{A})}=\mathcal{A}$. The proof is finished. 
Corollary 3.1. Suppose that $\operatorname{RR}(\mathcal{J})=0$. If $\mathcal{B}$ satisfies one of the following conditions, then $\mathcal{A}$ has (APD):

(1) $\mathcal{B}$ has (APD) and every projection in $\mathcal{B}$ can be lifted to a projection in $\mathcal{A}$;

(2) the set of all one-sided invertible elements in $\mathcal{B}$ is dense in $\mathcal{B}$, especially, $\operatorname{tsr}(\mathcal{B})=1$ or $\mathcal{B}$ is simple purely infinite;

(3) $\mathcal{B}$ is extremally rich and $\operatorname{ext}(\mathcal{B}) \subset \pi(\operatorname{Pis}(\mathcal{A}))$.

Proof. (1) Let $v \in \operatorname{Ais}(\mathcal{B})$. It is well known that if $v^{*} v$ or $v v^{*}$ can be lifted to a projection in $\mathcal{A}$, then there is $w \in \operatorname{Pis}(\mathcal{A})$ such that $\pi(w)=v$. Therefore $\mathcal{A}$ has (APD) by Theorem 1.1 and Proposition 2.4 .

(2) Since the isometry (or coisometry or unitary) in $\mathcal{B}$ can be lifted to a partial isometry in $\mathcal{A}$ and $\mathrm{Ais}(\mathcal{B})$ consists of isometries or coisometries in $\mathcal{B}$ by assumption, it follows from Theorem 1.1 and Proposition 2.4 that $\mathcal{A}$ has (APD).

If $\operatorname{tsr}(\mathcal{B})=1$, then $\operatorname{Ais}(\mathcal{B})=U(\mathcal{B})$; if $\mathcal{B}$ is a simple purely infinite $C^{*}$-algebra, then the set of all one-sided invertible elements is dense in $\mathcal{B}$ by 9 , Theorem 4.5 , Theorem 3.3]. Thus, $\mathcal{A}$ has (APD).

(3) Let $b \in \mathrm{Ws}_{0}(\mathcal{B})$ and $b=v|b|$ be the polar decomposition. Since $\overline{\mathcal{B}_{q}^{-1}}=B$, it follows from [3, Proposition 2.6] that there is $u \in \operatorname{ext}(\mathcal{B})$ such that $b=u|b|$. The assertion follows from the proof of Theorem 1.1 and Proposition 2.4

Corollary 3.1 shows that if $\operatorname{RR}(\mathcal{J})=0$ and $\operatorname{tsr}(\mathcal{B})=1$, then $\mathcal{A}$ has (APD). A natural problem is when $\mathcal{A}$ is extremally rich. We will use $K$-theory data to describe the extremal richness of $\mathcal{A}$ in Proposition 3.2 . Some $K$-theory notations used in the following may be found in [1] or 7 .

Let $\mathcal{E}$ be a $C^{*}$-algebra and let $\tau$ be a quasitrace on $\mathcal{E}$. Let $\tau_{n}$ be a quasitrace on the matrix algebra $\mathrm{M}_{n}(\mathcal{E})$ given by $\tau_{n}\left(\left(a_{i j}\right)_{n \times n}\right)=\sum_{i=1}^{n} \tau\left(a_{i i}\right)$. Thus $\tau$ induces a homomorphism $\tau_{*}: K_{0}(\mathcal{E}) \rightarrow \mathbb{R}$ defined by $\tau_{*}(x)=\tau_{n}(p)-\tau_{m}(q)$, for $x=[p]-[q] \in$ $K_{0}(\mathcal{E})$, where $p($ resp. $q)$ is a projection in $\mathrm{M}_{n}(\mathcal{E})\left(\right.$ resp. $\left.\mathrm{M}_{m}(\mathcal{E})\right)$.

Proposition 3.2. Let $\mathcal{J}$ be a $\sigma$-unital and simple $C^{*}$-algebra with $\mathrm{RR}(\mathcal{J})=0$ and $\operatorname{tsr}(\mathcal{J})=\operatorname{tsr}(\mathcal{A} / \mathcal{J})=1$. Suppose that there is a quasitrace $\tau$ on $\mathcal{J}$ such that for any projections $p_{1}, p_{2}$ in $\mathcal{J}$, if $\tau\left(p_{1}\right)<\tau\left(p_{2}\right)$, then $p_{1}$ is equivalent to a projection $q_{2} \leq p_{2}$ in $\mathcal{J}$. Then $\mathcal{A}$ is extremally rich iff $\operatorname{Ker} \tau_{*} \cap \operatorname{Im} \partial=\{0\}$, where $\left.\partial: K_{(} \mathcal{A} / \mathcal{J}\right) \rightarrow K_{0}(\mathcal{J})$ is the index map.

Proof. By Corollary $3.1(2), \mathcal{A}$ has $(\mathrm{APD})$. Since $\operatorname{tsr}(\mathcal{J})=\operatorname{tsr}(\mathcal{A} / \mathcal{J})=1$, it follows from [3, Corollary 6.3] that $\mathcal{A}$ is extremally rich iff for each $v \in U(\mathcal{A} / \mathcal{J})$, there is $w \in \operatorname{ext}(\mathcal{A})$ such that $\pi(w)=v$.

Now suppose that $\operatorname{Ker} \tau_{*} \cap \operatorname{Im} \partial=\{0\}$. Let $v$ be in $U(\mathcal{A} / \mathcal{J})$. Then there is $v \in \operatorname{Pis}(\mathcal{A})$ such that $\pi(v)=u$ and $\partial([u])=\left[1-v^{*} v\right]-\left[1-v v^{*}\right]$. So if $\tau\left(1-v^{*} v\right)=\tau\left(1-v v^{*}\right)$, then $\left[1-v^{*} v\right]=\left[1-v v^{*}\right]$. Since $\operatorname{tsr}(\mathcal{J})=1$, we can find $z_{0} \in \mathcal{J}$ such that $1-v^{*} v=z_{0}^{*} z_{0}, 1-v v^{*}=z_{0} z_{0}^{*}$. Put $w=u+z_{0}$. Then $w \in U(\mathcal{A})$ and $\pi(w)=u$. If $\tau\left(1-v^{*} v\right)>\tau\left(1-v v^{*}\right)$, there is $z_{1} \in \mathcal{J}$ such that $z_{1} z_{1}^{*}=1-v v^{*}, z_{1}^{*} z_{1} \leq 1-v^{*} v$ by the assumption of $\tau$. Put $w_{1}=v+z_{1}$. Then $w_{1}$ is a coisometry in $\mathcal{A}$ and $\pi\left(w_{1}\right)=u$; similarly, if $\tau\left(1-v^{*} v\right)<\tau\left(1-v v^{*}\right)$, we can find an isometry $w_{2}$ in $\mathcal{A}$ such that $\pi\left(w_{2}\right)=v$. Therefore, $\mathcal{A}$ is extremally rich.

Conversely, if $\operatorname{Ker} \tau_{*} \cap \operatorname{Im} \partial \neq\{0\}$, then there is $y \in K_{1}(\mathcal{A} / \mathcal{J})$ such that $\partial(y) \neq 0$ and $\tau_{*}(\partial(y))=0$. $\operatorname{tsr}(\mathcal{A} / \mathcal{J})=1$ implies that there is $u \in U(\mathcal{A} / \mathcal{J})$ such that $x=\partial(y)=\partial([u])$ (cf. [7, Theorem 3.1.11] or [10, Corollary 2.2]). Let $w \in \operatorname{Pis}(\mathcal{A})$ 
such that $\pi(w)=u$. Then $x=\left[1-w^{*} w\right]-\left[1-w w^{*}\right]$ in $K_{0}(\mathcal{J})$ and $\tau\left(1-w^{*} w\right)=$ $\tau\left(1-w w^{*}\right)$. Consequently, $w^{*} w \neq 1$ and $w w^{*} \neq 1$. Since $\mathcal{J}$ is simple, there exist $k_{1}, \cdots, k_{n} \in \mathcal{J}$ such that $1-w w^{*}=\sum_{i=1}^{n} k_{i}^{*}\left(1-w^{*} w\right) k_{i}$ by [7, Lemma 3.3.6]. Clearly, $w \notin \operatorname{ext}(\mathcal{A})$, so $\mathcal{A}$ is not extremally rich.

\section{ACKNOWLEDGMENT}

The author would like to thank Professor Larson and the referee for their helpful suggestions.

\section{REFERENCES}

[1] B. Blackadar, K-Theory for Operator Algebras (2nd ed.), Cambridge University Press, 1998. MR1656031 (99g:46104)

[2] L.G. Brown and G.K. Pedersen, $C^{*}$-algebras of real rank zero, J. Funct. Anal., 98 (1991), 131-149. MR:1120918 (92m:46086)

[3] L.G. Brown and G.K. Pedersen, On the geometry of the unit ball of a $C^{*}$-algebra, J. Reine Angew. Math., 469 (1995), 113-147. MR1363827 (96m:46101)

[4] U. Haagerup and M. Rørdam, $C^{*}$-algebras of unitary rank two, J. Operator Theory, 30 (1993), 161-171. MR.1302614 (95i:46086)

[5] R. Harte and M. Mbekhta, On generalized inverse in $C^{*}$-algebras, Studia Math., 103 (1992), 71-77. MR.1184103 (93i:46097)

[6] J. Jeong and H. Osaka, Extremally rich $C^{*}$-crossed products and the cancellation property, J. Aust. Math. Soc. (Ser. A), 64 (1998), 285-301. MR1623278 (99k:46097)

[7] H. Lin, An Introduction to the Classification of Amenable $C^{*}$-algebras, World Scientific, 2001. MR1884366 (2002k:46141)

[8] F. Perera, Extremal richness of multiplier and corona algebras of simple $C^{*}$-algebras with real rank zero, J. Operator Theory, 44 (2000), 413-431. MR.1794825 (2001m:46123)

[9] M. Rørdam, On the structure of simple $C^{*}$-algebras tensored with a UHF-algebra, J. Funct. Anal., 100 (1991), 1-17. MR1124289 (92m:46091)

[10] Y. Xue, The general stable rank in non-stable K-theory, Rocky Mount. J. Math., 30 (2000), 761-775. MR:1787012 (2001h:46125)

Department of Mathematics, East China Normal University, Shanghai 200062, PeoPLE'S REPUBLIC OF CHINA

E-mail address: yxue3486@hotmail.com 